\title{
Sub-Poissonian and anti-bunching criteria via majorization of statistics
}

\author{
Daniel G. Medranda and Alfredo Luis \\ Departamento de Óptica, Facultad de Ciencias Físicas, Universidad Complutense, \\ 28040 Madrid, Spain \\ E-mail: alluis@ucm.es
}

28 August 2018

\begin{abstract}
We use majorization and confidence intervals as a convenient tool to compare the uncertainty in photon number for different quantum light states. To this end majorization is formulated in terms of confidence intervals. As a suitable case study we apply this tool to sub- and super-Posissonian behavior and bunching and antibunching effects. We focus on the most significant classical and nonclassical states, such as Glauber coherent, thermal, photon number, and squeezed states. We show that majorization provides a more complete analysis that in some relevant situations contradicts the predictions of variance.

PACS numbers: 03.65.Ta, 42.50.Lc, 89.70.Cf, 02.50.-r
\end{abstract}

Keywords: Quantum uncertainty, Quantum optics, sub-Poissonian light, Majorization

\section{Introduction}

The most typical measure of uncertainty is variance, both in classical and quantum physics. However, variance is not the only measure of fluctuations nor the best behaved, as already pointed out for example in Refs. [1]. Some well-known disadvantages are, especially from a quantum perspective: (i) May fail when we move away from Gaussianlike statistics. In contrast to classical physics, non-Gaussian statistics is often the case in quantum physics. (ii) Puts too much emphasis on extremely unlikely outcomes. This may be a serious drawback in practical applications where the knowledge of the probability distribution is not complete. (iii) It cannot be used to derive Heisenberg-like uncertainty relations in finite-dimensional systems.

This has motivated the introduction of several measures of quantum fluctuations alternative to variance [2, 3, 4, 5, 6, , 7, 8]. Most of them have the form of an entropy, such as the Rényi and Tsallis entropies, that include the Shannon entropy as a particular case. At the very heart of these alternative entropic measures lies the concept of majorization 
since it is equivalent at once to the most popular entropic measures that satisfy the property of Schur-concavity.

In this work we address the majorization of photon-number statistics in quantum optics. We put a particular emphasis on the comparison with Poissonian statistics. The reason for this is due to the fact that the comparison of variances is the most classic test disclosing nonclassical behavior in quantum optics and plays a relevant role in quantum metrology [9]. The most significant advances presented in this contribution are:

(i) We show that majorization is equivalent to the unanimity of confidence intervals. Confidence intervals. They have been already used as a suitable measure of quantum uncertainty [8]. This extends the equivalence of majorization to other measures of uncertainty beyond entropies.

(ii) We show that majorization provides a much more stringent definition of sub- and super-Poissonian statistics, that may not coincide with the variance-based definition.

(iii) We extend the analysis to cover bunching and anti-bunching effects. Using variance as a measure of comparison they are equivalent to super- and sub-Poissonian statistics, respectively. We introduce a suitable criterion for bunching and anti-bunching in terms of majorization as a more complete statistical assessment. We show that from this perspective the equivalence with super- and sub-Poissonian statistics no longer holds and may provide a new practical criterion to disclose nonclassical behavior.

(iv) We apply this approach to the most frequent classical states, such as coherent and thermal states, as well as the most common nonclassical states, such as number states and squeezed states. All these states can be generated experimentally and have many practical applications.

The deeper statistical characterization provided by majorization can have interesting physical consequences in quantum physics and quantum optics in particular since fluctuations and uncertainty are a cornerstone of the quantum theory. For the sake of illustration let us focus on possible physical consequences in the area of quantum metrology.

One of the objectives of quantum metrology is to determine whether quantum fluctuations limit the precision in the detection of weak signals. Currently, most researchers believe that such limits exist under the form of Heisenberg limit [10]. However, such belief is based on variance estimators, so alternative approaches to quantum uncertainty may eventually be useful to overcome their metrological effects, as already suggested in Ref. [11. The probe states with sub-Poissonian photon statistics are crucial to reach the ultimate quantum resolution. Therefore, a more complete understanding of sub-Poissonian statistics may shed light on these limits and their eventual breaking.

Sub-Poissonian photon statistics is a paradigmatic nonclassical effect revealed by the comparison between variances. In this context, majorization is a comparison between complete distributions, so it may provide new tests of nonclassical behavior with a deep relation with previous nonclassical criteria introduced in terms of photon-number moments higher than variance [12, 13. Actually, majorization has been already used to 
derive higher-order criteria of nonclasssical behavior in Ref. [13].

Deep down, we can regard quantum-metrology limits as the consequence of the uncertainty relations satisfied by complementarity observables, such as time and energy. Uncertainty relations are typically expressed in terms of variance, so it may be interesting to investigate the consequences that might be derived from uncertainty relations expressed in terms of generalized entropies, or even via majorization such as in Ref. [14]. For example, there is no position-momentum uncertainty relation if uncertainty is assessed using some particular entropic measures [15]. Moreover, it has been shown that two observables can be complementary or not depending on the measure of fluctuations used [16], and nontrivial uncertainty measures can solve long standing debates on the enforcement of complementarity [17].

Finally, quantum metrology involves taking decisions with incomplete information. In such conditions it is preferable to work directly with probabilities rather than with their moments in order to obtain meaningful estimators of uncertainty.

In Sec. 2 we recall the concept of majorization, relating it with confidence intervals as suitable uncertainty measures, as well as most common photon statistics. We compare different photon statistics in Sec. 3. In Sec. 4 we extend these ideas to bunching and anti-bunching phenomena.

\section{Majorization, uncertainty and photon statistics}

In this section we just recall the concept of majorization, its relation with uncertainty measures, and the photon statistics of the most usual and interesting classical and nonclassical states.

\subsection{Majorization}

The distribution $p_{a}$ majorizes the distribution $p_{b}$, which will be expressed as $p_{b} \prec p_{a}$, when the following relation between the ordered partial sums $S_{N}$ holds, as illustrated in Fig. 1

$$
S_{N}\left(p_{b}^{\downarrow}\right)=\sum_{j=0}^{N} p_{b, j}^{\downarrow} \leq \sum_{k=0}^{N} p_{a, k}^{\downarrow}=S_{N}\left(p_{a}^{\downarrow}\right),
$$

for $N=0,1 \ldots \infty$, where the superscript $\downarrow$ on $p^{\downarrow}$ denotes the same distribution $p$ after a permutation of $p_{n}$ rearranging them in decreasing order

$$
p_{1}^{\downarrow} \geq p_{2}^{\downarrow} \geq \ldots \geq p_{j}^{\downarrow} \geq \ldots
$$

Majorization is a partial-ordering relation, so there are distributions that neither $p_{b} \prec p_{a}$ nor $p_{a} \prec p_{b}$.

\subsection{Uncertainty, entropies and confidence intervals}

Roughly speaking, if $p_{a}$ majorizes $p_{b}$ we have that $p_{b}$ is more disordered or more mixed than $p_{a}$. In other words, $p_{a}^{\downarrow}$ is more concentrated around its maximum. This can be made 


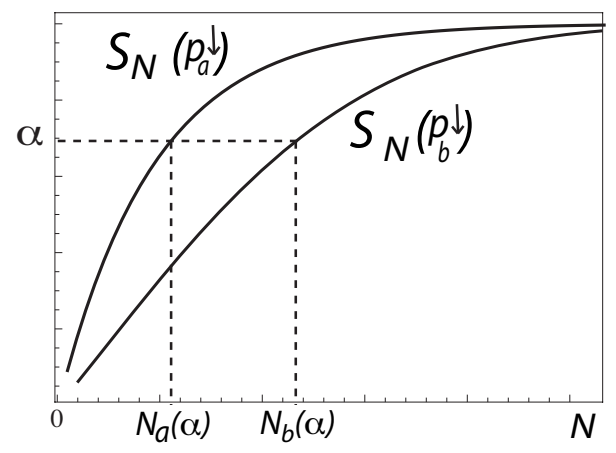

Figure 1. Relation between partial ordered sums $S_{N}$ and confidence intervals when the majorization $p_{b} \prec p_{a}$ holds.

more precise by noting that majorization is equivalent to a relation between statistics in terms of doubly stochastic matrices [2]. This means that $p_{b}$ can be obtained from $p_{a}$ by the addition of classical noise.

Thus majorization provides a very convenient measure of uncertainty. For example, the distribution with full certainty $p_{1}^{\downarrow}=1, p_{j \neq 1}^{\downarrow}=0$ has $S_{N}=1$ for all $N$, and majorizes all distributions. In the case of photo number, the states with full certainty are the photon-number states $|n\rangle$.

Whenever two distributions can be compared via majorization, the result is respected by the Schur-concave entropies, such as Tsallis and Rényi entropies:

$$
R_{q}\left(p_{i}\right)=\frac{1}{1-q} \ln \left(\sum_{i} p_{i}^{q}\right), \quad T_{q}\left(p_{i}\right)=\frac{1}{1-q}\left(\sum_{i} p_{i}^{q}-1\right),
$$

where $q \geq 0$ is the entropic index. The limiting case $\alpha \rightarrow 1$ is the Shannon entropy $T_{1}=R_{1}=-\sum_{i} p_{i} \log p_{i}$. Majorization implies that if $p_{b} \prec p_{a}$ then $H_{q}\left(p_{b}\right)>H_{q}\left(p_{a}\right)$ for all $q$ and $H=T, R$.

As an alternative and more direct connection of majorization with uncertainty measures, let us consider the confidence intervals $N(\alpha)$. They are defined as the minimum number of indices such that the partial sum up to $p_{N}^{\downarrow}$ comprises a given fraction $\alpha$ of the probability

$$
\sum_{j=0}^{N} p_{j}^{\downarrow} \geq \alpha, \quad N \geq N(\alpha) .
$$

When two distributions are comparable, $p_{b} \prec p_{a}$ is equivalent to saying that all confidence intervals of $p_{b}^{\downarrow}$ are larger than or equal to those of $p_{a}^{\downarrow}$ :

$$
p_{b} \prec p_{a} \longleftrightarrow N_{b}(\alpha) \geq N_{a}(\alpha) \forall \alpha .
$$

Otherwise, if the distributions are incomparable we will have $N_{b}(\alpha)>N_{a}(\alpha)$ and $N_{b}(\beta)<N_{a}(\beta)$ for some $\alpha, \beta$. 


\subsection{Photon number statistics}

Let us recall the photon-number statistics of the most significant classical and nonclassical states beyond the trivial case of photon-number states.

2.3.1. Coherent light The most celebrated light states regarded as classical in quantum optics are the Glauber coherent states. They have a Poissonian photon number distribution $p_{c, n}$

$$
p_{c, n}=\frac{\bar{n}^{n}}{n !} \exp (-\bar{n}), \quad \Delta^{2} n=\bar{n}
$$

This depends on a single parameter, the mean number of photons $\bar{n}$, that equals the variance $\Delta^{2} n$. Unfortunately, there is no suitable expression for $p_{c, n}^{\downarrow}$, nor for the partial sums $S_{N}\left(p_{c, n}^{\downarrow}\right)$. Nevertheless, in the case $\bar{n} \gg 1$ the Poissonian distribution can be well approximated by a Gaussian, treating $n$ as a continuous variable

$$
p_{c, n} \simeq p_{G, n}=\frac{1}{\Delta n \sqrt{2 \pi}} \exp \left[-(n-\bar{n})^{2} /\left(2 \Delta^{2} n\right)\right] .
$$

In this case $p_{G, n}^{\downarrow}$ can be easily obtained because of the full symmetry and monotonic behavior around the maximum at $\bar{n}$. The corresponding partial ordered sums are

$$
S_{N}\left(p_{G, n}^{\downarrow}\right)=\sum_{n=0}^{N} p_{G, n}^{\downarrow}=\frac{2}{\sqrt{\pi}} \int_{0}^{\frac{N}{\sqrt{2} \Delta n}} \mathrm{~d} u \exp \left(-u^{2}\right) .
$$

2.3.2. Thermal light Another basic and very common example of classical light are the thermal states, with photon-number statistics

$$
p_{t, n}=\frac{1}{\bar{n}+1}\left(\frac{\bar{n}}{\bar{n}+1}\right)^{n}, \quad \Delta^{2} n=\bar{n}(\bar{n}+1),
$$

that again depends on a single parameter. All thermal states are super-Poissonian $\Delta^{2} n>\bar{n}$. The probability distribution is in decreasing order from the start $p_{t, n}^{\downarrow}=p_{t, n}$, and the partial ordered sums can be easily obtained analytically as

$$
S_{N}\left(p_{t, n}^{\downarrow}\right)=\sum_{n=0}^{N} p_{t, n}^{\downarrow}=1-\left(\frac{\bar{n}}{\bar{n}+1}\right)^{N+1} .
$$

2.3.3. Squeezed light This is may be the best-known practical example of nonclassical light beyond photon-number states. Squeezed states are obtained from the vacuum $|0\rangle$ by a squeezing transformation $S(r)$ followed by a coherent displacement $D(R)$ as $D(R) S(r)|0\rangle$. For simplicity we will consider squeezing parameters such that the photonnumber distribution is [9]

$$
\begin{gathered}
p_{s, n}=\frac{\tanh ^{n} r}{2^{n} n ! \cosh r} \exp \left[-R^{2}(1-\tanh r)\right] \\
\times\left|H_{n}\left[\frac{\mathrm{i} R}{\sqrt{2}}\left(\sqrt{\tanh r}-\frac{1}{\sqrt{\tanh r}}\right)\right]\right|^{2},
\end{gathered}
$$


where $R$ represents the coherent contribution while the squeezing parameter $r$, with sign, is the squeezed part that represents the squeezing of quadrature fluctuations. The mean value and variance of the photon number are

$$
\bar{n}=R^{2}+\sinh ^{2} r, \quad \Delta^{2} n=R^{2} \mathrm{e}^{2 r}+\frac{1}{2} \sinh ^{2}(2 r) .
$$

They present sub- or super-Poissonian statistics depending on the $R, r$ values. In any case there is no simple form neither for $p_{s, n}^{\downarrow}$ nor for the ordered partial sums $S_{N}\left(p_{s, n}^{\downarrow}\right)$.

\subsection{Majorization applied to sub- and super-Posissonian statistics}

The idea of sub- and super-Posissonian statistics results from the comparison of the photon-number distribution $p_{n}$ of a given field state with a Poissonian statistics $p_{c, n}$ of the same mean number. This comparison has been always done in terms of variance. Majorization provides a much more stringent criterion as follows:

$$
p \prec p_{c} \equiv \text { under-Poissonian, } \quad p_{c} \prec p \equiv \text { over-Poissonian, }
$$

where in order to avoid confusions we have provided new different names. OverPoissonian means that the curve $S_{N}\left(p^{\downarrow}\right)$ is always above the curve $S_{N}\left(p_{c}^{\downarrow}\right)$ and vice versa for under-Poissonian. For Gaussian statistics over-Poissonian is equivalent to the sub-Poissonian and under-Poissonian equivalent to super-Poissonian. Otherwise, if the distributions are incomparable there can be no unambiguous claim about their relative photon-number uncertainty, and the result will depend on the particular entropy $H_{q}$ or confidence value $\alpha$ employed.

\section{Majorization relations}

Next we compare via majorization the photon-number distributions for the above classical and nonclassical states. When contrasting states from different families we will tend to compare states with the same mean number of photons. The reason to proceed in this way is that we are focusing on sub-Poissonian behavior, which implies comparison with a Poissonian distribution of the same mean.

\subsection{Coherent versus coherent}

It has been shown in Ref. [2] that any two Poisson distributions can be related through a doubly stochastic matrix in such a way that the distribution with smaller mean (this is smaller variance) majorizes the distributions with larger mean. Therefore, the order established by majorization coincides with the order established by variance and the distribution with smaller $\Delta n$ always majorizes the distribution with larger $\Delta n$. As illustration in Fig. 2 we have represented the ordered partial sums $S_{N}\left(p_{c, n}^{\downarrow}\right)$ as functions of $N$ for coherent states with different mean number of photons. This result is especially meaningful for small mean numbers. Otherwise, for $\bar{n} \gg 1$ the Gaussian approximation holds and majorization readily follows from variance after Eq. (8). 


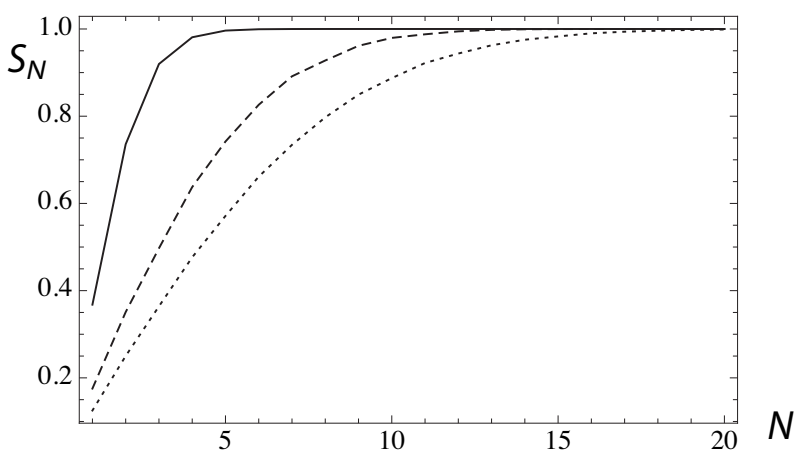

Figure 2. Ordered partial sums $S_{N}\left(p_{c, n}^{\downarrow}\right)$ as functions of $N$ for coherent states with mean number of photons (from top to bottom) $\bar{n}=1,5,10$. Throughout lines are drawn as guides for the eye.

\subsection{Thermal versus thermal}

An equivalent majorization relation consistent with variance follows for thermal light directly from relation $(10)$

$$
S_{N}\left(p_{t, a}^{\downarrow}\right)>S_{N}\left(p_{t, b}^{\downarrow}\right) \longleftrightarrow \Delta_{t, a} n<\Delta_{t, b} n .
$$

This case has been examined in more detail in Ref. [19]. In this case majorization may be pictured also as the result of added noise after Eq. (15) in Ref. [18] for example.

\subsection{Thermal versus coherent}

Using variance as a measure of comparison, the thermal light should always have larger uncertainty than coherent light for the same mean number. However we have found that they can be incomparable for small mean photon numbers, such as for $\bar{n}_{t}=\bar{n}_{c}=1.5$, as shown in Fig. 3. The ordered partial sums cross at a critical value $\tilde{\alpha} \simeq 0.80$ for $N(\tilde{\alpha}) \simeq 3$. Confidence intervals $N(\alpha<\tilde{\alpha})$ say that thermal light has less number fluctuations than coherent light, i. e., behaves as over-Poissonian light, while the confidence intervals $N(\alpha>\tilde{\alpha})$ say the opposite and the thermal behaves as underPoissonian. The transition occurs for a confidence interval that for Gaussian statistics would be between one and two standard deviations. On the other hand, for all cases examined with $\bar{n}_{c}=\bar{n}_{t} \gg 1$ the coherent states always majorize the thermal light, in accordance with variance.

When we remove the condition of equal mean, we can get incomparable distributions even in the case of large mean numbers $\bar{n}_{c}, \bar{n}_{t} \gg 1$. Moreover, in such a case we can find examples of full contradiction between majorization and variance, for example for $\bar{n}_{c}=100$ and $\bar{n}_{t}=10$. The variance of the thermal state is larger than the coherent, $\Delta_{t}^{2} n=110$ versus $\Delta_{c}^{2} n=100$, while the thermal almost majorizes the coherent, as shown in Fig. 4. Strictly speaking, the ordered partial sums cross at $\tilde{\alpha} \simeq 0.995$ for $N(\tilde{\alpha}) \simeq 57$

as shown in the inset of Fig. 4. However, the $99.5 \%$ of the photon-number statistics, and thus almost all confidence intervals $N(\alpha)$, support that the thermal state presents lesser 


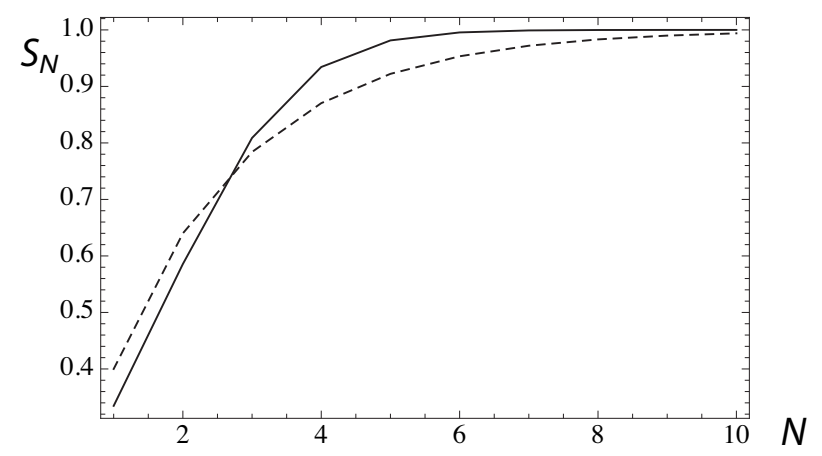

Figure 3. Ordered partial sums $S_{N}\left(p_{c, n}^{\downarrow}\right)$ and $S_{N}\left(p_{t, n}^{\downarrow}\right)$ as functions of $N$ for coherent (solid) and thermal (dashed) light with mean number of photons $\bar{n}_{t}=\bar{n}_{c}=1.5$.

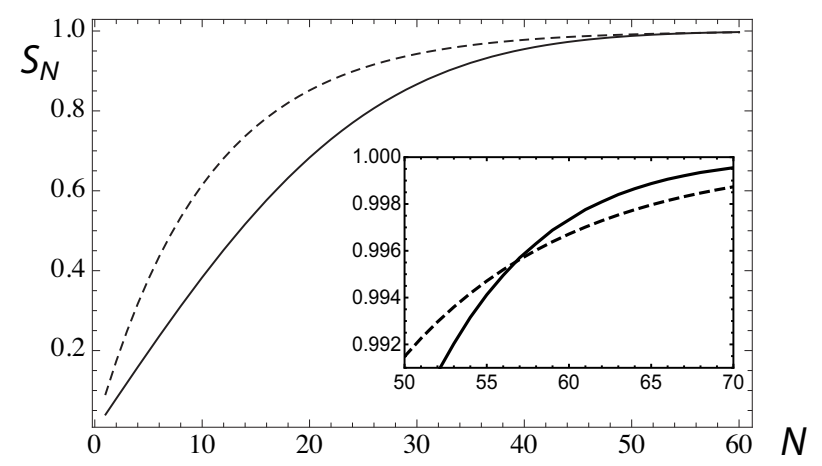

Figure 4. Ordered partial sums $S_{N}\left(p_{c, n}^{\downarrow}\right)$ and $S_{N}\left(p_{t, n}^{\downarrow}\right)$ as functions of $N$ for coherent (solid) and thermal (dashed) light with mean number of photons $\bar{n}_{t}=10$ and $\bar{n}_{c}=100$. The inset shows an extension and detail of the plot for larger $N$ revealing the crossing of the curves.

uncertainty than coherent light, contradicting variance. This result clearly illustrates how variance overestimates outcomes with extremely small probability, such as $0.5 \%$ in our case.

\subsection{Squeezed versus squeezed and coherent}

In the case of squeezed states we will consider always the same mean number of photons $\bar{n}$ for all states, but different number variance for some particular combinations of $R$ and $r$ Eq. (12). We have found incomparable statistics when contrasting sub-Poissonian states, when comparing super-Poissonian states, as well as when comparing them with Poissonian light.

An example of incomparable states is illustrated in Fig. 5 where we have plotted the ordered partial sums $S_{N}\left(p_{n}^{\downarrow}\right)$ for three different light states with $\bar{n}=6$ and variances $\Delta_{\text {sub }}^{2} n=0.6 \bar{n}$ (dashed), $\Delta_{\text {super }}^{2} n=14 \bar{n}$ (dotted), and $\Delta_{c}^{2} n=\bar{n}$ (solid). The superPoissonian case is actually the squeezed vacuum $R=0$. We can appreciate that both squeezed states are incomparable and the partial sums cross at $\tilde{\alpha} \simeq 0.60$ for $N(\tilde{\alpha}) \simeq 2.6$. 


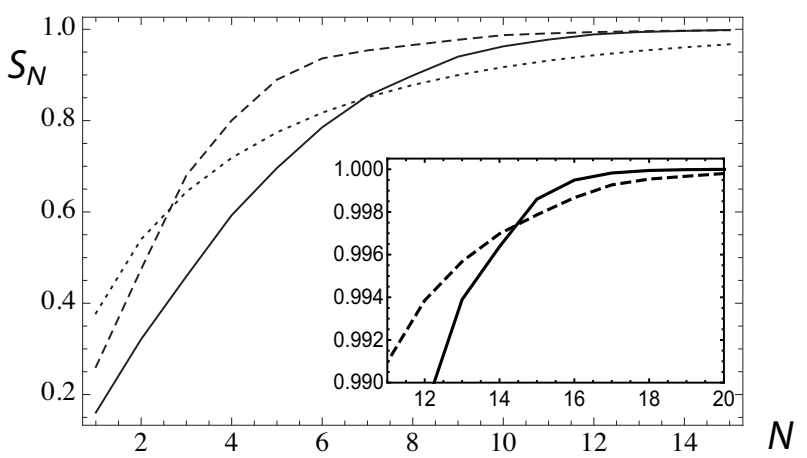

Figure 5. Ordered partial sums for variance-like super-Poissonian squeezed state with $\Delta_{\text {super }}^{2} n=14 \bar{n}$ (dotted), sub-Poissonian squeezed state with $\Delta_{\text {sub }}^{2} n=0.6 \bar{n}$ (dashed) and coherent light $\Delta_{c}^{2} n=\bar{n}$ (solid) as functions of $N$ with $\bar{n}=6$. The inset shows an extension and detail of the plot for larger $N$ values revealing the crossing of the sub-Poissonian and coherent curves.

Moreover the coherent and super-Poissonian states are incomparable as well and the partial sums cross at $\tilde{\alpha}=0.85$ for $N(\tilde{\alpha}) \simeq 7$.

On the other hand, it seems in the main plot of Fig. 5 that the sub-Poissonian light (dashed line) majorizes the coherent light (solid line). Strictly speaking, this is not exactly the case if we look closer to large $N$ values, displayed in the inset in Fig. 5, since the partial sums cross at $\tilde{\alpha}=0.997$ for $N(\tilde{\alpha}) \simeq 14$. This might be related to the characteristic photon-number oscillations of squeezed light. Nevertheless the fact that the states are incomparable has no practical consequences since it relies on extremely fine details of the photon-number distribution that will be hidden by experimental uncertainty.

\section{Bunching and anti-bunching}

A simple and direct practical observation of the sub- and super-Poissonian statistics is provided by the anti-bunching and bunching phenomena [20]. This is investigated by mixing the field state $\rho$ with vacuum at a $50 \%$ lossless beam splitter (see Fig. 6). At the output beams two photodetectors register the output photon numbers $n_{1}$ and $n_{2}$. In these conditions it can be easily seen that

$$
\Delta n_{1} \Delta n_{2}=\overline{n_{1} n_{2}}-\bar{n}_{1} \bar{n}_{2}=\frac{1}{4}\left(\Delta^{2} n-\bar{n}\right) .
$$

Therefore, the sub- and super-Poissonian character is reflected unambiguously on the sign of $\Delta n_{1} \Delta n_{2}$ : super-Poissonian light produces positive correlations $\Delta n_{1} \Delta n_{2}>0$, which is known as bunching, while sub-Poissonian light produces negative correlations $\Delta n_{1} \Delta n_{2}<0$, known as anti-bunching.

We translate to this framework the ideas around majorization in order to provide statistical characterizations beyond $\Delta n_{1} \Delta n_{2}$. This turns out to be quite simple if we focus on the number sum $n_{+}=n_{1}+n_{2}$ and number difference $n_{-}=n_{1}-n_{2}$ since for 


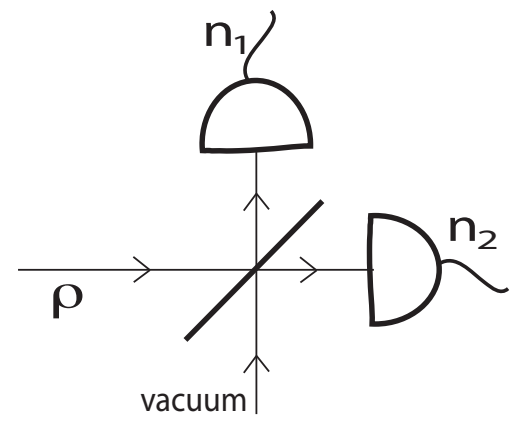

Figure 6. Arrangement for the observation of the bunching and anti-bunching effects.

these variables we have

$$
\Delta n_{1} \Delta n_{2}=\frac{1}{4}\left(\Delta^{2} n_{+}-\Delta^{2} n_{-}\right),
$$

so that bunching is equivalent to $\Delta n_{+}>\Delta n_{-}$while anti-bunching means $\Delta n_{+}<\Delta n_{-}$. Because of number conservation and the vacuum input all registered photons $n_{1}, n_{2}$ come from the state $\rho$ and no photon is lost. Therefore, the number sum coincides with the number variable $n_{+}=n$ for all $\rho$. Then, after Eqs. (15) and (16) we have always $\Delta^{2} n_{-}=\bar{n}$, so that the variable $n_{+}$carries the number statistics of the state examined while the variable $n_{-}$provides a Poissonian reference.

After Eq. (16) we can introduce a more complete criterion beyond variance via a majorization comparison between the distributions for the number sum $p_{+}$and number difference $p_{-}$. As we have just said, the $p_{+}$distribution coincides with the number distribution of the input estate

$$
p_{+, n}=p_{n}=\langle n|\rho| n\rangle,
$$

while for $p_{-}$we have

$$
p_{-, m}=\sum_{n=|m|}^{\infty} p_{n} \frac{1}{2^{n}}\left(\begin{array}{c}
n \\
\frac{n+m}{2}
\end{array}\right),
$$

and we note that when the number sum $n$ is odd or even, the number difference $m$ is also odd or even. Then we can introduce the following criterion

$$
p_{+} \prec p_{-} \equiv \text { clustering, } \quad p_{-} \prec p_{+} \equiv \text { anti-clustering, }
$$

where in order to avoid confusions with the variance-based bunching we will use the name clustering. Thus clustering means $N_{+}(\alpha) \geq N_{-}(\alpha)$ for all $\alpha$ and is the majorization counterpart of bunching. Otherwise, if the distributions are incomparable there can be no unambiguous claim, and the result will depend on the particular entropy $H_{q}$ or confidence value $\alpha$ employed to assess the uncertainty.

Note that the clustering criterion makes no reference whatsoever to the statistics of coherent states, since both $p_{ \pm}$in Eqs. (17) and (18) emerge exclusively from the number statistics of the state being examined. After Eq. (15) the variance-based bunching and anti-bunching are equivalent to super- and sub-Poissonian statistics, respectively. 


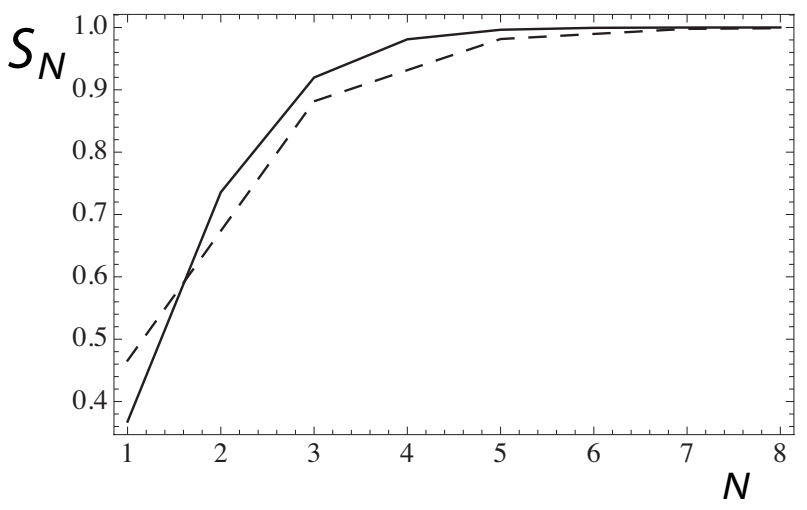

Figure 7. Ordered partial sums for the number-sum distribution $p_{+}$(solid line) and the number difference distribution $p_{-}$(dashed line) for a coherent state with $\bar{n}=1$.

However this is not the case of the majorization-based clustering and anti-clustering with regard to under- and over-Poissonian statistics. Over-Poissonian means $p_{c} \prec p$ while anti-clustering $p_{-} \prec p$. There seems to be no logical connection between these conditions since $p_{-}$in general is not Poissonian $p_{c}$.

Next we look for any relation between anti-clustering and nonclassicality that might parallel the well known role played by sub-Poissonian statistics as a nonclassical criterion. There is a simple connection if we focus on classical models where vacuum means a wave of zero amplitude. In such a case, after the $50 \%$ beam splitter in Fig. 6 the intensities recorded at both detectors are necessarily equal, regardless of the amplitude of the signal wave. This implies that the $p_{-, m}$ distribution has all probability concentrated in a single outcome, say $m=0$, and therefore $p_{-, m}$ will majorize any other distribution. Thus, the anti-clustering criterion in Eq. (19) would be a nonclassical effect.

As a first and readily example we may consider a coherent state. There is no simple expression for $p_{-, m}$ in Eq. (18) so we resort to a numerical comparison. We have found that for large mean numbers, say $\bar{n} \geq 5$, the partial sums of $p_{-, m}^{\downarrow}$ and $p_{+, n}^{\downarrow}$ are almost indistinguishable. On the other hand, for small mean numbers the situation is slightly different as shown in Fig. 7 for $\bar{n}=1$. The distributions turn out to be incomparable while all the partial sums of $p_{-, m}^{\downarrow}$ except the first one are below the sums of $p_{+, n}^{\downarrow}$. The case $\bar{n}=1$ is where the difference of partial sums is maximal. Therefore, the classicality criterion $p_{+} \prec p_{-}$is not satisfied in this case, which otherwise is an example of classical light according to most of other criteria, with the exceptions in Ref. [21]. This example also illustrates the lack of equivalence between clustering and Poissonian behavior.

Furthermore, we have applied criteria (19) to the incomparable cases in Fig. 3 and Fig. 5 always obtaining majorization relations that agree with the comparison of their variances. We have obtained more interesting results examining the super-Poissonian squeezed state with $\bar{n}=6$ and $\Delta^{2} n=2 \bar{n}$. Despite variance, this state displays an effective over-Poissonian behavior (less uncertainty than a coherent state of the same $\bar{n}$ ) for confidence intervals up to $\alpha \simeq 0.9$, as illustrated in Fig. 8 . This agrees with 


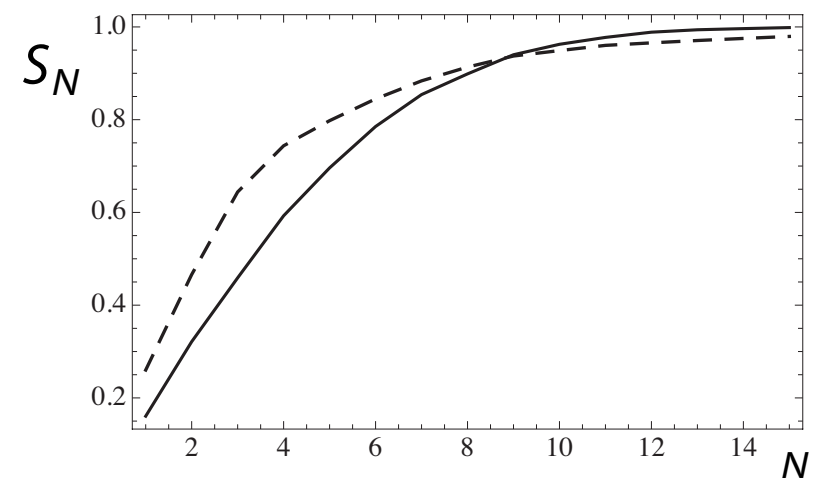

Figure 8. Ordered partial sums for the photon-number distribution of coherent squeezed state with super-Poissonian variance $\Delta^{2} n=2 \bar{n}$ (dashed) and coherent light $\Delta^{2} n=\bar{n}$ (solid) as functions of $N$ with $\bar{n}=6$. There is a clear effective over-Poissonian behavior (less uncertainty than a coherent state of the same $\bar{n}$ ) for confidence intervals up to $\alpha \simeq 0.9$. This contradicts the comparison of their variances.

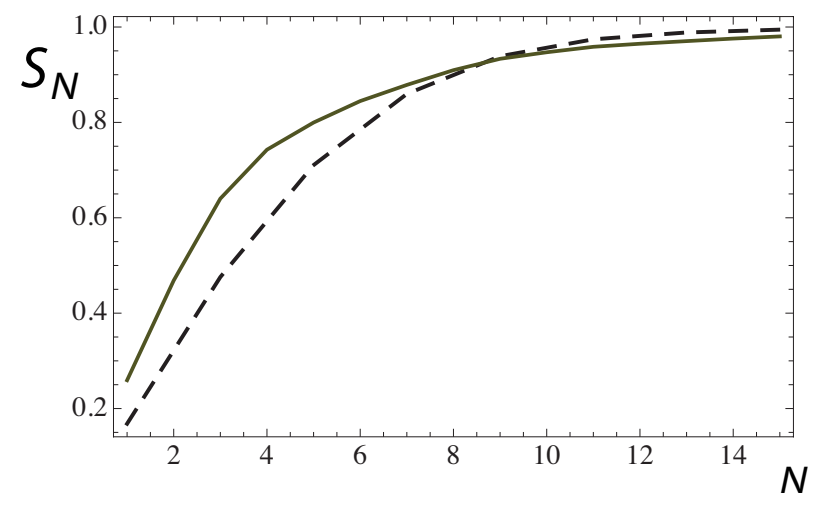

Figure 9. Ordered partial sums for the number-sum distribution $p_{+}$(solid line) and the number difference distribution $p_{-}$(dashed line) for the coherent squeezed state with super-Poissonian variance $\Delta^{2} n=2 \bar{n}$ and $\bar{n}=6$. There is a clear effective anticlustering effect (less uncertainty in the number sum than in the number difference) for confidence intervals up to $\alpha \simeq 0.9$. This contradicts the conclusions derived from variance.

the ordered partial sums for $p_{ \pm}$in Fig. 9 that show that this state presents clear anticlustering (less uncertainty in the number sum than in the number difference) for all confidence intervals with $\alpha$ values up to $\alpha=0.9$.

In order to find further results contradicting the conclusions derived from variance let us consider the following mix of one-photon $|1\rangle$ and thermal $\rho_{t}$ states

$$
\rho=\xi|1\rangle\left\langle 1\left|+(1-\xi) \rho_{t}, \quad \rho_{t}=\frac{1}{1+\bar{n}_{t}} \sum_{n=0}^{\infty}\left(\frac{\bar{n}_{t}}{1+\bar{n}_{t}}\right)^{n}\right| n\right\rangle\langle n|,
$$

where $\xi$ is a mixing parameter and $\bar{n}_{t}$ is the mean number of the thermal state $\rho_{t}$. The complete state $\rho$ which has a mean number of photons of $\bar{n}=\xi+(1-\xi) \bar{n}_{t}$. For example for $\bar{n}=2$ and $\xi=0.9$ the state $\rho$ is clearly super-Poissonian $\Delta^{2} n=11 \bar{n}$. However 


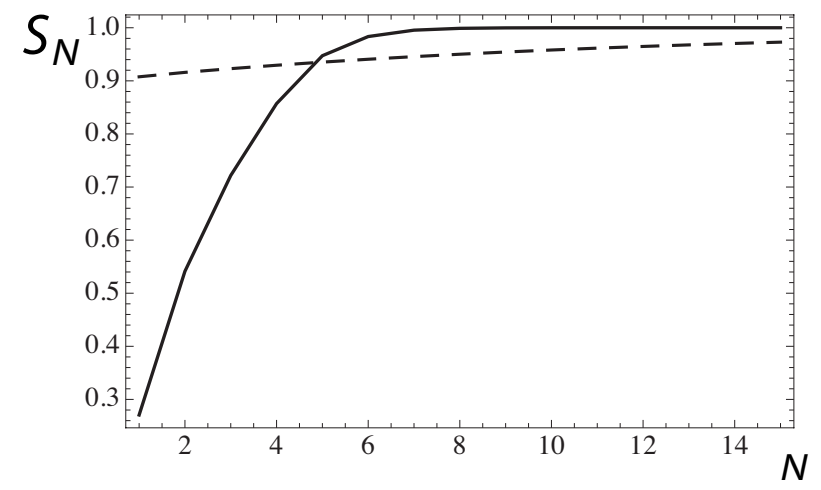

Figure 10. Ordered partial sums for the photon times thermal state 20 with $\bar{n}=2$ and $\xi=0.9$ in dashed line and a coherent state of the same mean number in solid line.

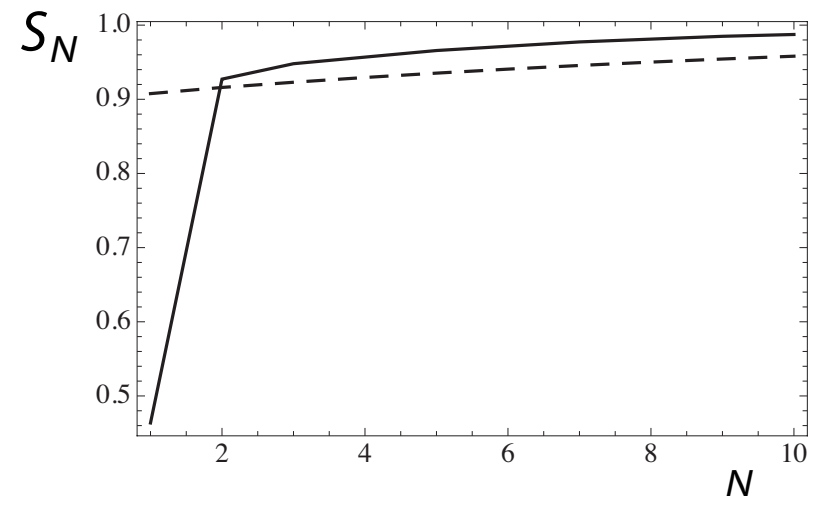

Figure 11. Ordered partial sums for the number-sum distribution $p_{+}$(solid line) and the number difference distribution $p_{-}$for the photon times thermal state 20 with $\bar{n}=2$ and $\xi=0.9$.

the whole statistics tell the full opposite. This is a consequence of the fact that the number distribution is strongly concentrated around $n=1$, with $p_{1}=0.91$, so for most practical purposes this works as a single-photon state, that is, over-Poissonian and anticlustered. This is clearly reflected in Fig. 10 when comparing with a coherent state of the same mean number. The states are incomparable, but when referring to confidence intervals enclosing up to $90 \%$ of the statistics $\alpha \leq 0.9$ the state behaves effectively as over-Poissonian (less fluctuations than the coherent state) in spite of $\Delta^{2} n=11 \bar{n}$. This is again the effect on variance of large photon numbers with negligible probabilities.

Regarding anti-clustering, the ordered partial sums of the distributions $p_{ \pm}$are represented in Fig. 11. They show that the states are incomparable. But again, for confidence intervals with $\alpha \leq 0.9$ the state behaves as anti-clustered (less uncertainty in the number sum than in the number difference), in contradiction with the predictions of variance. In particular the probability that only one detector registers light - this is the probability of $n_{1} n_{2}=0$-is above $92 \%$. 


\section{Conclusions}

We have compared the photon statistics of the most relevant classical and nonclassical states from the perspective of majorization and confidence intervals. We have focused on the ideas of sub- and super-Poissonian statistics as well as the bunching and antibunching effects.

As the most relevant results, we have found: (i) There are several interesting and common examples of incomparable states. (ii) Majorization may contradict variance. (iii) There is a majorization counterpart of bunching which is logically independent of Poissonian statistics. Let us briefly comment on these results.

The information provided by majorization is valuable even when dealing with incomparable states. Previous works have found that different entropies lead to contradictory conclusions, where the minimum uncertainty states with one measure are the maximum uncertainty states of the other [22]. Majorization clearly explains this result showing that these contradictions arise because we are dealing with incomparable states [23].

Majorization can contradict variance because the former involves the complete statistics (that is, moments of all orders of the distribution), while variance only involves the first two moments.

In any case, majorization will always provide a deeper knowledge of the situation than focusing exclusively on the variance. We do not propose to adopt majorization as a better way of comparing statics, but as another tool to understand quantum features. The use of one measure of the other will ultimately depend on the particular tasks addressed and the characteristic of the data available.

\section{Acknowledgments}

A. L. acknowledges support from Projects No. FIS2012-35583 of the Spanish Ministerio de Economía y Competitividad and CAM research consortium QUITEMAD+ S2013/ICE-2801.

\section{References}

[1] Hilgevoord J 2002 Am. J. Phys. 70 983-983

Lawrence G N 1994 Laser Focus World 30 109-114

[2] Marshall A W, Olkin I and Arnold B C 2011 Inequalities: Theory of Majorization and Its Applications (New York: Springer)

[3] Aczel J and Daroczy Z 1975 On Measures of Information and Their Characterization (New York: Academic Press)

[4] Ruch E, Schranner R and Seligman Th H 1978 J. Chem. Phys. 69 386-392

Herbut F and Ivanović I D 1982 J. Phys. A 15 1775-1783

[5] Rényi A On the measures of entropy and information, Proc. 4th Berkeley Symp. on Mathematics and Statistical Probability, University of California Press 1961 Berkeley pp. 547-561

[6] Tsallis C 1988 J. Stat. Phys. 52 479-487

[7] Beck Ch 2009 Contemporary Physics 50 495-510 
[8] Uffink J B M and Hilgevoord J 1984 Phys. Lett. A 105 176-178

Uffink J B M and Hilgevoord J 1985 Found. Phys. 15 925-944

Lévy-Leblond J M 1985 Phys. Lett. A 111 353-355

Hall M J W 1993 J. Mod. Opt. 40 809-824

[9] Scully M O and Zubairy M S 1997 Quantum Optics (Cambridge: Cambridge University Press)

[10] Giovannetti V, Lloyd S and Maccone L 2004 Science 306 1330-1336

Giovannetti V, Lloyd S and Maccone L 2011 Nature Photonics 5 222-229

Tsang M 2012 Phys. Rev. Lett. 108230401

Hall M J W and Wiseman H M 2012 New J. Phys. 14033040

[11] Luis A and Rodil A 2013 Phys. Rev. A 87034101

[12] Peřina J 1991 Quantum Statistics of Linear and Nonlinear Optical Phenomena (Dordrecht: Kluwer)

Peřina Jr J and Peřina J 2000 Progress in Optics 41 361-419

Pathak A and Garcia M 2006 Appl. Phys. B 84 479-484

[13] Lee C T 1990 Opt. Lett. 15 1386-1388

Lee C T 1990 Phys. Rev. A 41 1721-1723

[14] Partovi H 2011 Phys. Rev. A 84052117

Puchała Z, Rudnicki Łand Życzkowski K 2013 J. Phys. A 46272002

Friedland S, Gheorghiu V and Gour G 2013 Phys. Rev. Lett. 111230401

[15] Zozor S, Portesi M and Vignat C 2008 Physica A 387 4800-4808.

[16] Luis A 2002 Phys. Rev. Lett. 88230401

[17] Luis A and Sánchez-Soto L L 1998 Phys. Rev. Lett. 81 4031-4035

[18] Hall M J W 1994 Phys. Rev. A 50 3295-3303

[19] Canosa N, Rossignoli R and Portesi M 2006 Physica A 368 435-441

Canosa N, Rossignoli R and Portesi M 2006 Physica A 371 126-129

[20] Mandel L and Wolf E 1995 Optical Coherence and Quantum Optics (Cambridge: Cambridge University Press)

[21] Vaccaro J A 1995 Phys. Rev. A 52 3474-3488

Johansen L M 2004 Phys. Lett. A 329, 184-187

Luis A 2006 Phys. Rev. A $\mathbf{7 3} 063806$

[22] Luis A 2011 Phys. Rev. A 84034101

Matía-Hernando P and Luis A 2012 Phys. Rev. A 86052106

[23] Luis A, Bosyk G M and Portesi M 2015 arXiv:1501.06667 [quant-ph] 